\section{ROBUST VARIABLE-FIDELITY OPTIMIZATION OF MICROWAVE FILTERS USING CO-KRIGING AND TRUST REGIONS}

\author{
Slawomir Koziel, ${ }^{1}$ Leifur Leifsson, ${ }^{1}$ Ivo Couckuyt, ${ }^{2}$ \\ and Tom Dhaene ${ }^{2}$ \\ Surrogate Modeling Lab, Department of Information Technology, \\ Gent University_-IBBT, B-9050 Gent, Belgium; Corresponding \\ author: koziel@ru.is \\ 2 Engineering Optimization \& Modeling Center, School of Science \\ and Engineering, Reykjavik University, Menntavegur 1, 101 \\ Reykjavik, Iceland
}

Received 3 August 2012

\begin{abstract}
This work introduces a variable-fidelity optimization methodology for simulation-driven design optimization of filters. Our approach is based on electromagnetic (EM) simulations of different accuracy controlled by the mesh density. A Kriging interpolation model (the surrogate) is created using sampled low-fidelity EM data and optimized to approximately locate the optimum of the high-fidelity EM model of the filter. This initial surrogate is subsequently improved by blending in the high-fidelity data accumulated during the optimization process using the co-Kriging technique. The algorithm convergence is ensured by embedding it into the trust region framework. The operation and performance of our method is demonstrated using three filter design cases. (C) 2012 Wiley Periodicals, Inc. Microwave Opt Technol Lett 55:765-769, 2013; View this article online at wileyonlinelibrary.com. DOI: $10.1002 /$ mop.27447
\end{abstract}

Key words: filter design; electromagnetic simulation; surrogate modeling, Kriging; co-Kriging; computer-aided design; surrogate-based optimization; trust region framework

\section{INTRODUCTION}

Electromagnetic (EM) simulation is a primary tool used in the design of microwave structures. In many cases, the initial designs can be obtained using simplified models, e.g., analytical formulas or equivalent circuits. However, in order to satisfy given performance requirements, these initial designs have to be further finetuned. Often, the adjustment of geometry/material parameters is realized through repetitive simulations or parameter sweeps (usually, one parameter at a time). This approach is tedious and does not guarantee optimum results. It is therefore desirable to automate the design closure by employing numerical optimization techniques and making the design cycle shorter and more robust.

Unfortunately, automated EM-simulation-driven design using conventional optimization techniques (e.g., gradient-based ones) may be impractical. This is because accurate EM analysis is computationally expensive and large number of such analyses may be necessary to find an optimal design. Reduction of the design cost is possible through the use of surrogate models, i.e., cheap and yet reasonably accurate representations of the structure under consideration. A popular way of creating the surrogate is by approximating high-fidelity EM simulation data using, e.g., Kriging [1], support vector regression [2], or neural networks [3]. However, decent accuracy of the model can only be ensured if the design space is sampled sufficiently dense. In practice, hundreds or even thousands of samples may be necessary. This can be justified for multiple use library models but not for one-time optimization of a given structure.

Simulation-driven design can be realized more efficiently through surrogate-based techniques exploiting physics-based models. The most popular methods include space mapping (SM) [4] and simulation-based tuning [5]. Unfortunately, these and other similar methods lack robustness necessary for their full automation, or their implementation is too complex to be widely accepted by engineering community. Recently, adjoint sensitivity techniques [6] have become available through commercial EM solvers (e.g., [7]), which permits obtaining derivative information at little or no extra computational cost. Adjoint sensitivities can substantially speed up conventional gradient-based optimization $[8,9]$. Nevertheless, the development of robust algorithms that exploit this technology is a relatively complex task for an inexperienced user. On the other hand, even when using derivative information, a gradient-based routine may still require considerable number of EM analyses.

In this work, a robust algorithm for microwave design optimization is introduced that exploits the co-Kriging methodology [10]. The co-Kriging technique is a convenient tool to combine coarsediscretization EM simulations (much cheaper than the high-fidelity ones) and limited number of high-fidelity EM data into a single surrogate model which is fast to compute. The high-fidelity data is accumulated during the optimization process, so no extra expensive EM simulations are necessary. The iterative updating and reoptimization of the co-Kriging surrogate is embedded into the trust region framework to ensure good convergence properties. Several microstrip filter examples are considered for demonstration purposes with satisfactory designs obtained at the cost of a few highfidelity EM simulations in each case.

\section{DESIGN OPTIMIZATION OF FILTERS USING CO-KRIGING AND TRUST REGIONS}

Design closure of many microwave devices, including filters, is performed using high-fidelity EM simulations, which is a necessary, although tedious, task often performed using repetitive parameter sweeps. In this section, we formulate the design task as a nonlinear minimization problem, as well as describe the proposed solution approach that exploits variable fidelity EM simulations, namely, Kriging and co-Kriging interpolation and the trust region framework. The main purpose of our methodology is to reduce the computational cost of the design process while making it as robust as possible. We also formulate the complete optimization algorithm. Its numerical verification is postponed until Section 3.

\subsection{Design Problem Formulation}

Let $\boldsymbol{R}_{f} \in \boldsymbol{R}^{m}$ denote the response vector of a high-fidelity model of the filter structure of interest, typically, $S$-parameters evaluated at $m$ different frequencies, $\boldsymbol{x} \in R^{n}$ be a vector of design variables (e.g., geometry parameters), and $U$ be a given objective function which is formulated so that a better design corresponds to a lower value of $U\left(\boldsymbol{R}_{f}(\boldsymbol{x})\right)$. The filter design problem can be formulated as a nonlinear minimization task of the form

$$
\boldsymbol{x} * \in \arg \min _{x} U\left(R_{f}(\mathbf{x})\right)
$$

where $x^{*}$ is the design variable vector corresponding to an optimum design.

\subsection{Surrogate-Based Optimization}

The high-fidelity model is typically obtained using accurate CPUintensive EM simulation. Due to the high computational cost, direct optimization of $\boldsymbol{R}_{f}$ (using, e.g., gradient-based algorithms) may be impractical as it requires a large number of model evaluations. In this work, we focus on a surrogate-based approach, where the direct solution to Eq. (1) is replaced by an iterative scheme

$$
\boldsymbol{x}^{(i+1)}=\arg \min _{\left\|x-x^{(i)}\right\| \leq \delta^{(i)}} U\left(R_{s}^{(i)}(x)\right)
$$


that produces a sequence $\boldsymbol{x}^{(i)}$ of approximate solutions to Eq. (1). Here, $\boldsymbol{R}_{s}^{(i)}$ is a surrogate model at iteration $i$, which is a computationally cheap representation of $\boldsymbol{R}_{f}$. In order to ensure convergence of the algorithm (2), the surrogate model optimization is constrained to the vicinity of the current design $\boldsymbol{x}^{(i)}$ defined by a trust region radius $\delta^{(i)}$ [11]. The value of the trust region radius is updated based on the gain ratio defined as follows

$$
\rho^{(i)}=\frac{U\left(R_{f}\left(\boldsymbol{x}^{(i+1)}\right)\right)-U\left(R_{f}\left(\boldsymbol{x}^{(i)}\right)\right)}{U\left(R_{s}^{(i)}\left(\boldsymbol{x}^{(i+1)}\right)\right)-U\left(R_{s}^{(i)}\left(\boldsymbol{x}^{(i)}\right)\right)}
$$

$\rho^{(i)}$ measures the quality of the objective function improvement prediction made by the surrogate model. The trust region radius is increased if $\rho^{(i)}$ is sufficiently large (i.e., the prediction made by the surrogate model can be trusted) and decreased if it is too small. If $U\left(\boldsymbol{R}_{f}\left(x^{(i+1)}\right)\right) \geq U\left(\boldsymbol{R}_{f}\left(x^{(i)}\right)\right)$, the new design $x^{(i+1)}$ is rejected and the search starts again from $\boldsymbol{x}^{(i)}$ using the reduced $\delta^{(i)}$.

\subsection{Surrogate Modeling Using Kriging and Co-Kriging}

In this work, the surrogate model is created using the co-Kriging methodology that allows us to combine EM simulations of different fidelity. Densely sampled low-fidelity EM data (referred to as $\boldsymbol{R}_{c}$ ) is used to create the initial Kriging interpolation model, which is subsequently enhanced by a limited number of high-fidelity EM data points using co-Kriging. The high-fidelity data is obtained by evaluating $\boldsymbol{R}_{f}$ at all previous iteration points $\boldsymbol{x}^{(i)}$, which has to be done anyway for verification purposes. By using the co-Kriging technique, we are able to create a surrogate model that is almost as accurate as the high-fidelity model but substantially cheaper, because the majority of training data comes from the low-fidelity EM simulations.

Kriging is a popular technique for interpolating deterministic noise-free data [12]. We will denote by $X_{B . c}=\left\{\boldsymbol{x}_{c}{ }^{1}, \boldsymbol{x}_{c}{ }^{2}, \ldots\right.$, $\left.\boldsymbol{x}_{c}^{N . c}\right\}$ the training set and by $\boldsymbol{R}_{\boldsymbol{c}}\left(X_{B . c}\right)$ the associated coarse-discretization model responses. The Kriging interpolation model with a constant mean $\alpha$ is defined as

$$
R_{S . K R}(\boldsymbol{x})=\alpha+r(\boldsymbol{x}) \cdot \Psi^{-1} \cdot\left(R_{c}\left(X_{B . C}\right)\right)-\mathbf{1} \alpha
$$

where $\mathbf{1}$ is a column vector of ones. The coefficient vector $\alpha$ is determined by generalized least squares. $r_{i}(\boldsymbol{x})=\psi\left(\boldsymbol{x}, \boldsymbol{x}_{c}{ }^{i}\right)$, for $i=$ $1 \ldots N_{\mathrm{c}}$, is a vector of correlations, and $\Psi_{i, j}=\psi\left(\boldsymbol{x}_{c}{ }^{i}, \boldsymbol{x}_{c}{ }^{j}\right)$, for $i$ $=1 \ldots N_{\mathrm{c}}$ and $j=1 \ldots N_{\mathrm{c}} . \Psi$ is a correlation matrix. The parameters $\theta_{1}, \ldots, \theta_{n}$ of the correlation function $\psi(\cdot, \cdot)$ are identified by a Maximum Likelihood Estimation (MLE).

Co-Kriging interpolation [10] combines the fine data $\boldsymbol{R}_{f}$ and the coarse data $\boldsymbol{R}_{c}$ to make an accurate prediction. In particular, the autoregressive co-Kriging model of Kennedy et al. [10] is used.

First, a Kriging model $\boldsymbol{R}_{S . K R c}$ of the coarse data $\left(X_{B . c}, \boldsymbol{R}_{c}\left(X_{B . c}\right)\right)$ is constructed and, subsequently, a second Kriging model $\boldsymbol{R}_{s . K R d}$ is constructed on $\left(X_{B . f}, \boldsymbol{R}_{d}\right)$, where $\boldsymbol{R}_{d}=$ $\boldsymbol{R}_{\mathrm{f}}\left(X_{B . f}\right)-\rho \cdot \boldsymbol{R}_{\mathrm{c}}\left(X_{B . f}\right)$. The parameter $\rho$ of the second Kriging model $\boldsymbol{R}_{S . K R d}$ is included in the MLE. The response values $\boldsymbol{R}_{C}\left(X_{B . f}\right)$ (or an approximation thereof) are easily obtained by evaluating the first Kriging model $\boldsymbol{R}_{s . K R c}$, namely, $\boldsymbol{R}_{c}\left(X_{B . f}\right)$. $\boldsymbol{R}_{s . K R c}\left(X_{B . f}\right)$. Lastly, the two Kriging models are combined to construct the co-Kriging interpolant

$$
R_{s}(\boldsymbol{x})=M \alpha+r(\boldsymbol{x}) \cdot \Psi_{-1} \cdot\left(R_{d}-F \alpha\right)
$$

where the block matrices $M, F, r(\boldsymbol{x})$ and $\Psi$ can be written as a function of the two Kriging models $\boldsymbol{R}_{S . K R c}$ and $\boldsymbol{R}_{\text {s.KRd }}$ :

$$
\begin{gathered}
r(\boldsymbol{x})=\left[\rho \cdot \sigma_{c}^{2} \cdot r_{c}(\boldsymbol{x}), \rho^{2} \cdot \sigma_{c}^{2} \cdot r_{c}\left(\boldsymbol{x}, X_{B_{f}}\right)+\sigma_{d}^{2} r_{d}(\boldsymbol{x})\right] \\
\Psi=\left[\begin{array}{cc}
\sigma_{d}^{2} \Psi_{c} & \rho \cdot \sigma_{c}^{2} \Psi_{c}\left(X_{B \cdot C}, X_{B \cdot f}\right) \\
0 & \rho^{2} \cdot \sigma_{c}^{2} \Psi_{c}\left(X_{B \cdot f}, X_{B \cdot f}\right)+\sigma_{c}^{2} \cdot \Psi_{d}
\end{array}\right] \\
F=\left[\begin{array}{cc}
\mathbf{1} & 0 \\
\rho \cdot \mathbf{1} & \mathbf{1}
\end{array}\right], \quad M=\left[\begin{array}{ll}
\rho & 1
\end{array}\right]
\end{gathered}
$$

where $\sigma_{c}^{2}$ and $\sigma_{d}^{2}$ are process variances, while $\Psi_{c}(\cdot, \cdot)$ and $\Psi_{d}(\cdot, \cdot)$ denote correlation matrices of two datasets with the optimized $\theta_{1}, \ldots, \theta_{n}$ parameters and correlation function of the Kriging models $\boldsymbol{R}_{S . K R c}$ and $\boldsymbol{R}_{s . K R d}$, respectively. In this work, the exponential correlation function is used for both Kriging models, i.e., $\psi\left(x, x^{\prime}\right)=\exp \left(\Sigma_{k=1, \ldots, n}-\theta_{k} \mid \boldsymbol{x}^{k}-\boldsymbol{x}^{\prime k}\right)$.

\subsection{Optimization Flow}

The proposed design optimization procedure can be summarized as follows (here, $\boldsymbol{x}^{\text {init }}$ is the initial design):

1. Starting from $\boldsymbol{x}^{\text {init }}$, optimize $\boldsymbol{R}_{c}$ to find $\boldsymbol{x}^{(0)}$-the initial design for the co-Kriging optimization;

2. Sample $\boldsymbol{R}_{c}$ in the vicinity of $\boldsymbol{x}^{(0)}$; evaluate $\boldsymbol{R}_{c}$ to obtain the base set $\left(X_{B . c}, \boldsymbol{R}_{c}\left(X_{B . c}\right)\right)$

3. Set the iteration index $i=0$;

4. Create a co-Kriging model $\boldsymbol{R}_{S}^{(i)}$ as in Eq. (5) using $\left(X_{B . c}, \boldsymbol{R}_{c}\left(X_{B . c}\right)\right)$ and $\left(X_{B . f}, \boldsymbol{R}_{f}\left(X_{B . f}\right)\right)$ with $X_{B . f}=\left\{\boldsymbol{x}^{(0)}, \ldots, \boldsymbol{x}^{(i)}\right\}$;

5. Find the new design $\boldsymbol{x}^{(i+1)}$ by optimizing $\boldsymbol{R}_{s}^{(i)}$ as in Eq. (2);

6. Calculate gain ratio $\rho^{(i)}=\left[U\left(\boldsymbol{R}_{f}\left(\boldsymbol{x}^{(i+1)}\right)\right)-U\left(\boldsymbol{R}_{f}\left(\boldsymbol{x}^{(i)}\right)\right)\right] /$ $\left[U\left(\boldsymbol{R}_{s}^{(i)}\left(\boldsymbol{x}^{(i+1)}\right)\right)-U\left(\boldsymbol{R}_{S}^{(i)}\left(\boldsymbol{x}^{(i)}\right)\right)\right]$

7. If $\rho^{(i)}>\rho_{\text {incr }}$, set $\delta^{(i+1)}=\delta^{(i)} \cdot m_{\text {incr, }}$, elseif $\rho^{(i)}<\rho_{\text {decr }}$, set $\delta^{(i+1)}=\delta^{(i)} / m_{\mathrm{decr}}$, else $\delta^{(i+1)}=\delta^{(i)}$;

8. If $\left\|\boldsymbol{x}^{(i)}-\boldsymbol{x}^{(i-1)}\right\| \varepsilon$ or $\delta^{(i)}<\varepsilon$, terminate;

9. If $U\left(\boldsymbol{R}_{f}\left(\boldsymbol{x}^{(i+1)}\right)\right)<U\left(\boldsymbol{R}_{f}\left(\boldsymbol{x}^{(i)}\right)\right)$, set $i=i+1$ and go to 4 , else set $\delta^{(i)}=\delta^{(i+1)}$ and go to 5 ;

In order to reduce the number of training samples necessary to set up the co-Kriging model, it is only created in the vicinity of the $\boldsymbol{R}_{c}$ optimum, which is the best approximation of the optimal design we can get at a low cost. The size of the vicinity is typically 5 to $20 \%$ of the design space. The initial co-Kriging surrogate is created using only one evaluation of $\boldsymbol{R}_{f}$ and then updated using the designs obtained by optimizing the surrogate. As co-Kriging is an interpolative model for the expensive data, we have $\boldsymbol{R}_{s}^{(i)}\left(\boldsymbol{x}^{(k)}\right)=\boldsymbol{R}_{f}\left(\boldsymbol{x}^{(k)}\right)$ for $k=0, \ldots, i$, so that the surrogate accuracy improves in the vicinity of the expected optimum upon the algorithm convergence.

The parameters used to update the trust region radius (cf. Step 7 of the algorithm) are the following: $\rho_{\text {incr }}=0.5, \rho_{\text {decr }}=0.01$, $m_{\text {incr }}=2, m_{\text {decr }}=5$. The termination condition parameter $\varepsilon$ is set to $10^{-3}$. As indicated above, the algorithm is terminated either upon convergence or if the trust region size is sufficiently small.

\section{DESIGN EXAMPLES}

In this section, a numerical verification of the co-Kriging-based filter optimization procedure is provided. We consider three examples of microstrip filter structures and investigate the performance of the proposed algorithm both with respect to the quality of the final design obtained in the course of optimization and the computational cost of the design process.

\subsection{Half-Wavelength Stepped Impedance Resonator Filter}

A half-wavelength stepped impedance resonator (SIR) bandpass filter [13], shown in Figure 1, is considered. The EM simulations are carried out in FEKO [14] for both the high-fidelity model $\boldsymbol{R}_{f}$ (total mesh number is 1090 , simulation time $22 \mathrm{~min}$ ) 


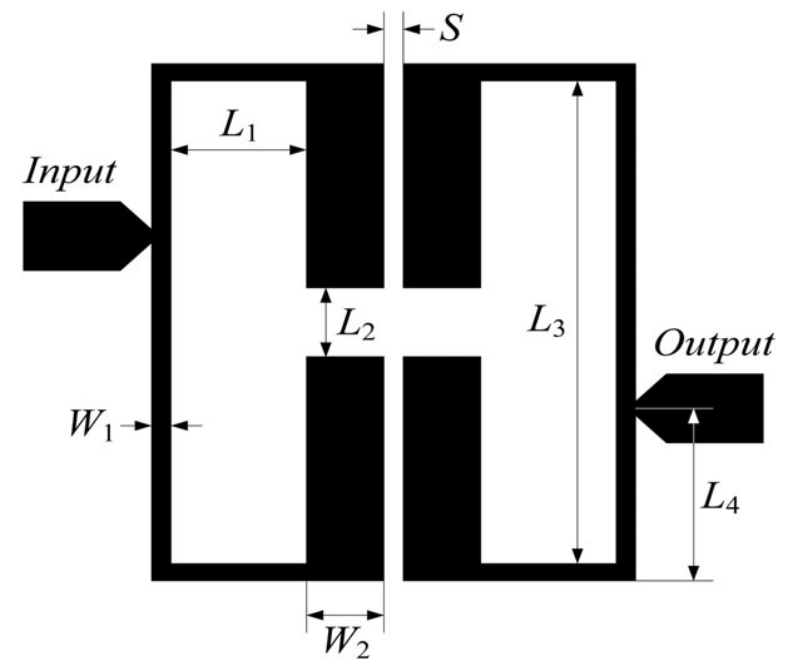

Figure 1 Geometry of the half-wavelength SIR bandpass filter [13]

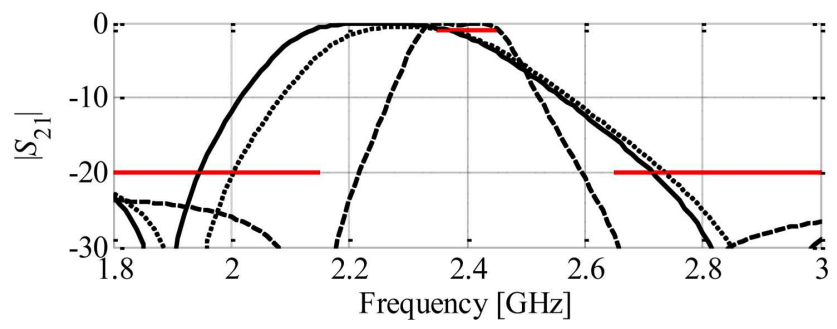

Figure 2 Responses of the half-wavelength SIR bandpass filter: low(- - -) and high-fidelity (-) models at the initial design $\boldsymbol{x}^{\text {init }}$. [Color figure can be viewed in the online issue, which is available at wileyonlinelibrary.com]

and the low-fidelity model $\boldsymbol{R}_{c}$ (total mesh number 128, simulation time $30 \mathrm{~s})$. The design variables are $\boldsymbol{x}=\left[\begin{array}{lllll}L_{1} & L_{2} & L_{3} & L_{4} & S\end{array} W_{1}\right.$ $\left.W_{2}\right]^{T}$. The design specifications are $\left|S_{21}\right| \geq-1 \mathrm{~dB}$ for $2.35 \mathrm{GHz}$ to $2.45 \mathrm{GHz}$, and $\left|S_{21}\right| \leq-20 \mathrm{~dB}$ for $1.5 \mathrm{GHz}$ to $2.15 \mathrm{GHz}$ and $2.65 \mathrm{GHz}$ to $3.2 \mathrm{GHz}$. The initial design is $\boldsymbol{x}^{\text {init }}=\left[\begin{array}{ll}2.5 & 1.5 \\ 11.6\end{array}\right.$ $\left.\begin{array}{llll}2.95 & 0.328 & 0.305 & 1.613\end{array}\right]^{T} \mathrm{~mm}$. Optimization of the filter was performed using the co-Kriging-based algorithm of Section 2.

First, the low-fidelity model $\boldsymbol{R}_{c}$ is optimized directly, at the cost of 100 evaluations, to yield $\boldsymbol{x}^{(0)}=\left[\begin{array}{llll}2.96 & 1.54 & 10.41 & 3.76\end{array}\right.$ $\left.\begin{array}{lll}0.59 & 0.35 & 1.81\end{array}\right]^{T} \mathrm{~mm}$. Figure 2 shows the responses of $\boldsymbol{R}_{c}$ and $\boldsymbol{R}_{f}$ at $\boldsymbol{x}^{\text {init }}$, and the response of $\boldsymbol{R}_{c}$ at $\boldsymbol{x}^{(0)}$. The co-Kriging surrogate is then created in the region $\left[\boldsymbol{x}^{(0)}-\delta, \boldsymbol{x}^{(0)}+\delta\right]$, with $\delta=[0.5$ $\left.\begin{array}{llllll}0.1 & 0.2 & 0.2 & 0.1 & 0.1 & 0.1\end{array}\right]^{T} \mathrm{~mm}$, using $100 \boldsymbol{R}_{c}$ samples. The coKriging optimization process yields the design $\boldsymbol{x}^{(6)}=\left[\begin{array}{ll}2.68 & 1.56\end{array}\right.$ $\left.\begin{array}{lllll}10.38 & 3.72 & 0.61 & 0.38 & 1.85\end{array}\right]^{T} \mathrm{~mm}$ after six iterations. Figures 3

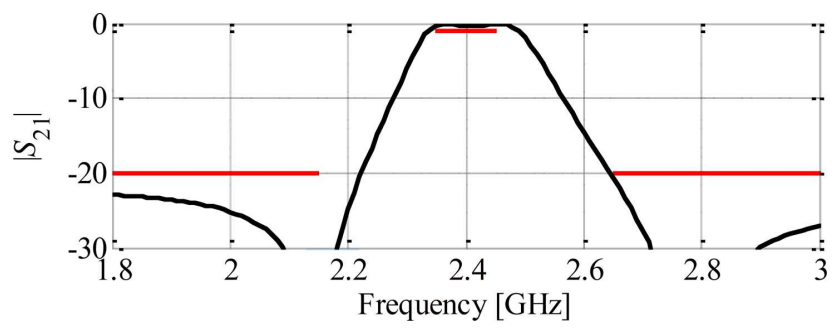

Figure 3 Responses of the half-wavelength SIR bandpass filter: $\boldsymbol{R}_{\mathrm{c}}$ (..) and $\boldsymbol{R}_{\mathrm{f}}\left(-\right.$ - ) at $\boldsymbol{x}^{(0)}$ as well as $\boldsymbol{R}_{f}(-)$ at the final design $\boldsymbol{x}^{(6)}$. [Color figure can be viewed in the online issue, which is available at wileyonlinelibrary.com]

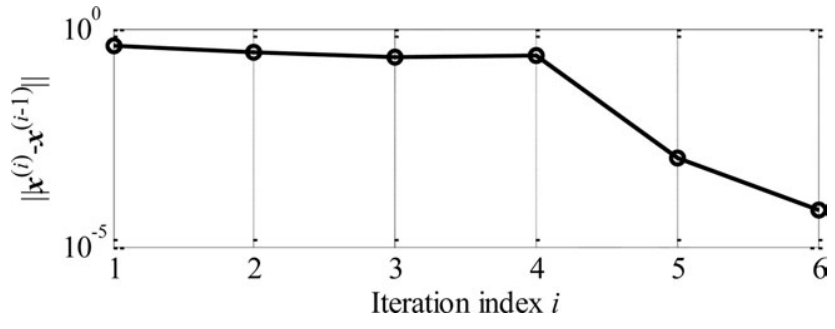

Figure 4 Convergence of the co-Kriging-based optimization algorithm for the half-wavelength SIR bandpass filter

TABLE 1 Half-wavelength SIR Filter: Optimization Results

\begin{tabular}{lccc}
\hline \multirow{2}{*}{$\begin{array}{l}\text { Algorithm } \\
\text { Component }\end{array}$} & $\begin{array}{c}\text { Number of } \\
\text { Model Evaluations }\end{array}$ & \multicolumn{2}{c}{ CPU Time } \\
\cline { 3 - 4 } & $200 \times \boldsymbol{R} c$ & 100 & 4.5 \\
Evaluation of $\boldsymbol{R}_{c}$ & $9 \times \boldsymbol{R} f$ & 198 & 9.0 \\
Evaluation of $\boldsymbol{R}_{f}$ & N/A & 298 & $\mathbf{1 3 . 5}$ \\
Total cost & &
\end{tabular}

Includes 100 evaluations necessary to optimize $\boldsymbol{R}_{c}$ and 100 evaluations to set up the co-Kriging model.

Excludes $\boldsymbol{R}_{f}$ evaluation at the initial design.

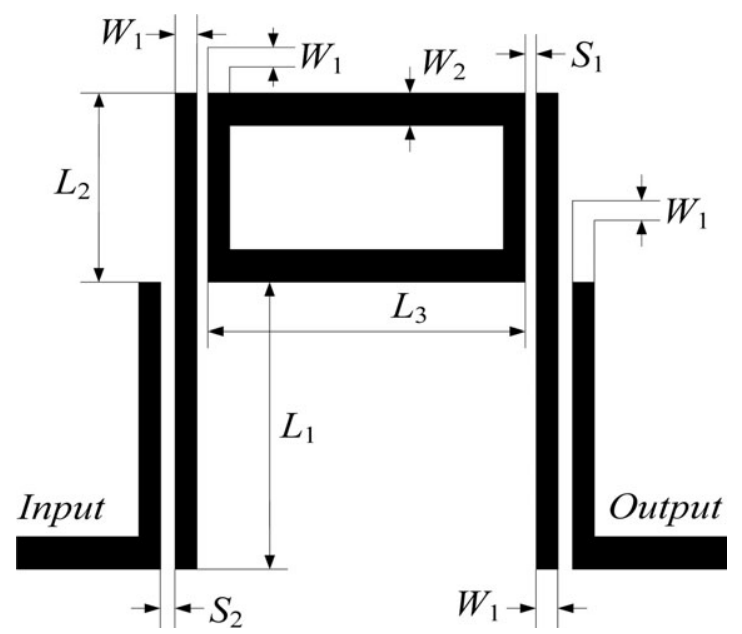

Figure 5 Geometry of the fourth-order ring resonator bandpass filter [15]

and 4 show the high-fidelity model response at $\boldsymbol{x}^{(6)}$ and the convergence plot, respectively. The total design cost (Table 1) corresponds to about 13 evaluations of $\boldsymbol{R}_{f}$.

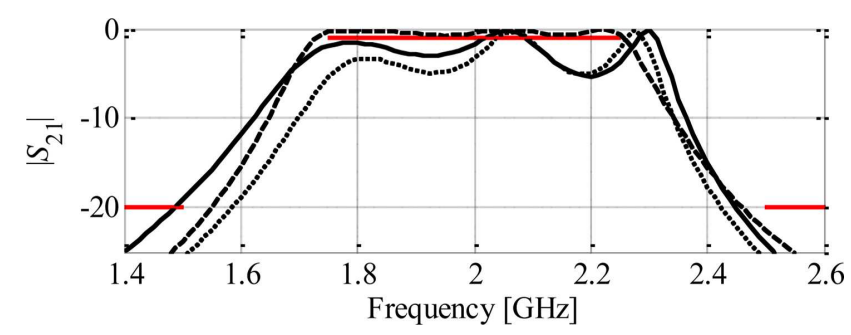

Figure 6 Responses of the fourth-order ring-resonator bandpass filter: low- (- - -) and high-fidelity (-) models at the initial design $x^{i n i t}$. [Color figure can be viewed in the online issue, which is available at wileyonlinelibrary.com] 
TABLE 2 Fourth-order Ring Resonator Filter: Optimization Results

\begin{tabular}{lccr}
\hline \multirow{2}{*}{$\begin{array}{l}\text { Algorithm } \\
\text { Component }\end{array}$} & $\begin{array}{c}\text { Number of } \\
\text { Model Evaluations }\end{array}$ & $\begin{array}{c}\text { Absolute } \\
(\mathrm{min})\end{array}$ & $\begin{array}{r}\text { Relative } \\
\text { to } \boldsymbol{R} f\end{array}$ \\
\cline { 3 - 4 } Evaluation of $\boldsymbol{R}_{c}{ }^{\mathrm{a}}$ & $180 \times \boldsymbol{R} c$ & 90 & 4.5 \\
Evaluation of $\boldsymbol{R}_{f}^{\mathrm{b}}$ & $10 \times \boldsymbol{R} f$ & 200 & 10.0 \\
Total cost & N/A & 290 & 14.5 \\
\hline
\end{tabular}

${ }^{a}$ Includes 80 evaluations necessary to optimize $\boldsymbol{R}_{c}$ and 100 evaluations to set up the co-Kriging model.

${ }^{\mathrm{b}}$ Excludes $\boldsymbol{R}_{f}$ evaluation at the initial design.

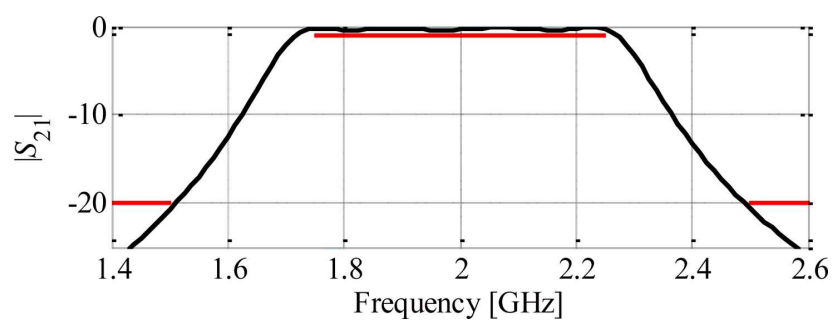

Figure 7 Responses of the fourth-order ring-resonator bandpass filter: $\boldsymbol{R}_{c}(\ldots)$ and $\boldsymbol{R}_{f}\left(-\right.$ - at $\boldsymbol{x}^{(0)}$ as well as $\boldsymbol{R}_{f}(-)$ at the final design $\boldsymbol{x}^{(7)}$. [Color figure can be viewed in the online issue, which is available at wileyonlinelibrary.com]

\subsection{Fourth-Order Ring Resonator Bandpass Filter}

We now consider the fourth-order ring resonator bandpass filter [15] shown in Figure 5, with the design parameters $\boldsymbol{x}=\left[\begin{array}{ll}L_{1} & L_{2}\end{array}\right.$ $\left.\begin{array}{lllll}L_{3} & S_{1} & S_{2} & W_{1} & W_{2}\end{array}\right]^{T} \mathrm{~mm}$. As before, FEKO [14] is employed to evaluate both $\boldsymbol{R}_{f}$ (total mesh number 978; evaluation time 20 min) and $\boldsymbol{R}_{c}$ (mesh number 174; evaluation time $30 \mathrm{~s}$ ). The design specifications are $\left|S_{21}\right| \geq-1 \mathrm{~dB}$ for $1.75 \mathrm{GHz} \leq \omega \leq$ $2.25 \mathrm{GHz}$, and $\left|S_{21}\right| \leq-20 \mathrm{~dB}$ for $1.0 \mathrm{GHz} \leq \omega \leq 1.5 \mathrm{GHz}$ and $2.5 \mathrm{GHz} \leq \omega \leq 3.0 \mathrm{GHz}$. The initial design is $x^{\text {init }}=[25$ $\left.\begin{array}{llllll}20 & 25 & 0.1 & 0.1 & 1.2 & 0.8\end{array}\right]^{T} \mathrm{~mm}$. The filter was optimized using the co-Kriging-based algorithm of Section 2.

An approximate optimum of $\boldsymbol{R}_{c}, \boldsymbol{x}^{(0)}=\left[\begin{array}{ll}22.9 & 20.426 .60 .12\end{array}\right.$ $\left.\begin{array}{lll}0.05 & 1.2 & 0.72\end{array}\right]^{T} \mathrm{~mm}$, is obtained at the cost of 80 evaluations. The responses are shown in Figure 6. The co-Kriging surrogate is created using $100 \boldsymbol{R}_{c}$ samples in the region $\left[x^{(0)}-\delta, x^{(0)}+\right.$ $\delta$ ] with $\delta=\left[\begin{array}{lllllll}0.5 & 0.5 & 0.5 & 0.05 & 0.05 & 0.1 & 0.1\end{array}\right]^{T} \mathrm{~mm}$. The final design, $\boldsymbol{x}^{(7)}=\left[\begin{array}{lllllll}22.5 & 20.2 & 26.5 & 0.169 & 0.061 & 1.16 & 0.72\end{array}\right]^{T} \mathrm{~mm}$, is found in seven iterations of the co-Kriging optimization process. The results are given in Figure 7 and Table 2. The total design cost corresponds to about 14 evaluations of $\boldsymbol{R}_{f}$.

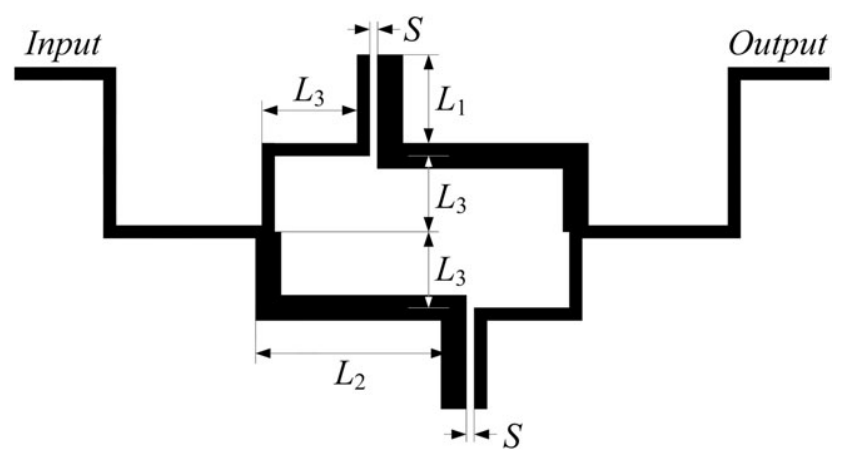

Figure 8 Second-order CCDBR filter: geometry [16]

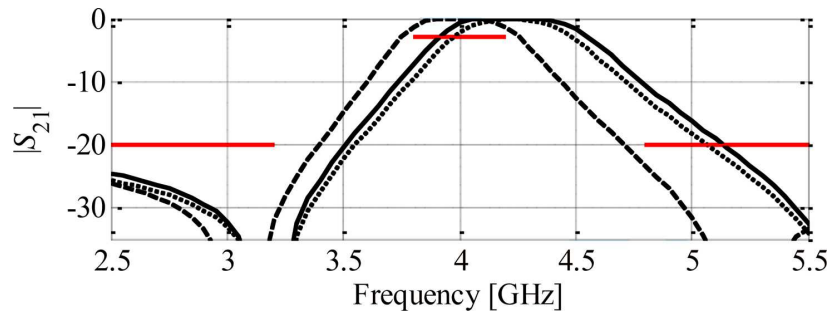

Figure 9 Responses of the CCDBR filter: low- (...) and high-fidelity (一) models at the initial design $x^{\text {init }}$, and the low-fidelity model at $x^{(0)}$ (- - ). [Color figure can be viewed in the online issue, which is available at wileyonlinelibrary.com]

TABLE 3 CCDBR Filter: Optimization Results

\begin{tabular}{lccc}
\hline \multirow{2}{*}{$\begin{array}{l}\text { Algorithm } \\
\text { Component }\end{array}$} & $\begin{array}{c}\text { Number of Model } \\
\text { Evaluations }\end{array}$ & $\begin{array}{c}\text { Absolute } \\
(\mathrm{min})\end{array}$ & $\begin{array}{c}\text { Relative } \\
\text { to } \boldsymbol{R} f\end{array}$ \\
\hline Evaluation of $\boldsymbol{R} c$ & $150 \times \boldsymbol{R} c$ & 90 & 1.5 \\
Evaluation of $\boldsymbol{R} f$ & $6 \times \boldsymbol{R} f$ & 180 & 6.0 \\
$\quad$ Total cost & $\mathrm{N} / \mathrm{A}$ & 270 & 7.5 \\
\hline
\end{tabular}

${ }^{\text {a }}$ Includes 50 evaluations necessary to optimize $\boldsymbol{R}_{c}$ and 100 evaluations to set up the co-Kriging model.

${ }^{\mathrm{b}}$ Excludes $\boldsymbol{R}_{f}$ evaluation at the initial design.

\subsection{Capacitively Coupled Dual-Behavior Resonator Microstrip Filter}

Now we consider the second-order capacitively coupled dualbehavior resonator (CCDBR) microstrip filter [16] shown in Figure 8, with the design variables $\boldsymbol{x}=\left[\begin{array}{llll}L_{1} & L_{2} & L_{3} & S\end{array}\right]^{T}$. Both high(total mesh number 1134; evaluation time $30 \mathrm{~min}$ ) and low-fidelity (mesh number 130; evaluation time $36 \mathrm{~s}$ ) models are simulated in FEKO [14]. The design specifications are $\left|S_{21}\right| \geq$ $-3 \mathrm{~dB}$ for $3.8 \mathrm{GHz} \leq \omega \leq 4.2 \mathrm{GHz}$, and $\left|S_{21}\right| \leq-20 \mathrm{~dB}$ for $2.0 \mathrm{GHz} \leq \omega \leq 3.2 \mathrm{GHz}$ and $4.8 \mathrm{GHz} \leq \omega \leq 6.0 \mathrm{GHz}$. The initial design is $\boldsymbol{x}^{(0)}=\left[\begin{array}{lll}3.0 & 5.0 & 1.0\end{array}\right]^{T} \mathrm{~mm}$.

An approximate optimum of $\boldsymbol{R}_{c}, \boldsymbol{x}^{(0)}=\left[\begin{array}{lll}3.2 & 4.96 & 1.2\end{array}\right]^{T} \mathrm{~mm}$, is obtained at the cost of 50 evaluations of $\boldsymbol{R}_{c}$. The responses are shown in Figure 8. The co-Kriging surrogate is setup in a region with the size $\delta=\left[\begin{array}{lll}0.25 & 0.25 & 0.25\end{array}\right]^{T} \mathrm{~mm}$. The optimized design, $\boldsymbol{x}^{(3)}=\left[\begin{array}{lll}3.2 & 4.98 & 1.22\end{array}\right]^{T} \mathrm{~mm}$, is found in three iterations. The results are given in Figure 9 and Table 3. The total design cost corresponds to less than eight evaluations of $\boldsymbol{R}_{f}$. Figure 10 shows the responses of the CCDBR filer.

\section{CONCLUSION}

A reliable and low-cost procedure for EM-simulation-driven design optimization of microwave filters has been described.

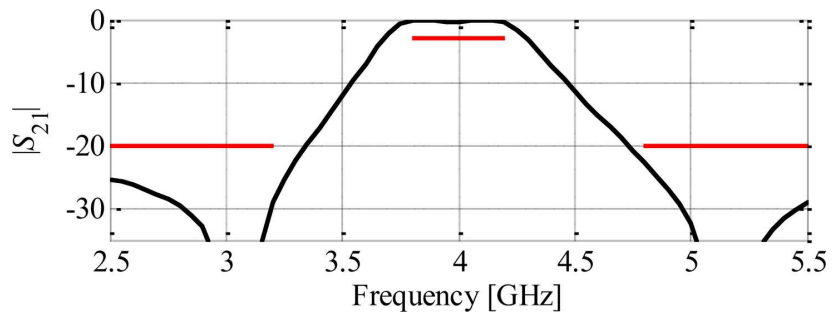

Figure 10 Responses of the CCDBR filter: high-fidelity model $\boldsymbol{R}_{f}$ at the final design found by the co-Kriging algorithm. [Color figure can be viewed in the online issue, which is available at wileyonlinelibrary.com] 
The procedure exploits variable-fidelity EM simulations that are combined into an accurate and computationally cheap surrogate model using co-Kriging interpolation. The surrogate is utilized in a prediction-correction scheme that is embedded into the trust region framework to ensure convergence. Three filter examples are considered to demonstrate performance of the proposed technique.

\section{ACKNOWLEDGMENTS}

This work was supported in part by the Icelandic Centre for Research (RANNIS) Grant 110034021. Ivo Couckuyt is funded by the Institute for the Promotion of Innovation through Science and Technology in Flanders (IWT-Vlaanderen).

\section{REFERENCES}

1. A.I.J. Forrester and A.J. Keane, Recent advances in surrogatebased optimization, Prog Aerospace Sci 45 (2009), 50-79.

2. G. Angiulli, M. Cacciola, and M. Versaci, Microwave devices and antennas modeling by support vector regression machines, IEEE Trans Magn 43 (2007), 1589-1592.

3. H. Kabir, Y. Wang, M. Yu, and Q.J. Zhang, High-dimensional neural-network technique and applications to microwave filter modeling, IEEE Trans Microwave Theory Tech 58 (2010), $145-156$

4. S. Koziel, Q.S. Cheng, and J.W. Bandler, Space mapping, IEEE Microwave Mag 9 (2008), 105-122.

5. D. Swanson and G. Macchiarella, Microwave filter design by synthesis and optimization, IEEE Microwave Mag 8 (2007), 55-69.

6. N. Georgieva, S. Glavic, M.H. Bakr, and J.W. Bandler, Feasible adjoint sensitivity technique for EM design optimization, IEEE Trans Microwave Theory Tech 50 (2002), 2751-2758.

7. CST Microwave Studio, ver. 2011, CST AG, Bad Nauheimer Str. 19, D-64289 Darmstadt, Germany, 2011.

8. D. Nair and J.P. Webb, Optimization of microwave devices using 3-D finite elements and the design sensitivity of the frequency response, IEEE Trans Magn 39 (2003), 1325-1328.

9. M.A. El Sabbagh, M.H. Bakr, and J.W. Bandler, Adjoint higher order sensitivities for fast full-wave optimization of microwave filters, IEEE Trans Microwave Theory Tech 54 (2006), 3339-3351.

10. M.C. Kennedy and A. O'Hagan, Predicting the output from complex computer code when fast approximations are available, Biometrika 87 (2000) 1-13.

11. S. Koziel, J.W. Bandler, and Q.S. Cheng, Robust trust-region space-mapping algorithms for microwave design optimization, IEEE Trans Microwave Theory Tech 58 (2010), 2166-2174.

12. D. Gorissen, K. Crombecq, I. Couckuyt, P. Demeester, and T. Dhaene, A surrogate modeling and adaptive sampling toolbox for computer based design, J Machine Learning Res 11 (2010), 2051-2055.

13. R. Ma, G. Han, X. Chen, L. Li, L. Han, and W. Zhang, Method for determining feed positions in coupled half-wavelength stepped impedance resonator bandpass filter, IET Microwaves Ant Prop 3 (2009), 856-862.

14. FEKO, Suite 6.0, EM Software \& Systems-S.A. (Pty) Ltd, 32 Techno Lane, Technopark, Stellenbosch, 7600, South Africa, 2010.

15. M.H.M. Salleh, G. Prigent, O. Pigaglio, and R. Crampagne, Quarter-wavelength side-coupled ring resonator for bandpass filters, IEEE Trans Microwave Theory Tech 56 (2008) 1, 156-162.

16. A. Manchec, C. Quendo, J.-F. Favennec, E. Rius, and C. Person, Synthesis of capacitive-coupled dual-behavior resonator (CCDBR) filters, IEEE Trans Microwave Theory Tech 54 (2006), 2346-2355.

(C) 2012 Wiley Periodicals, Inc.

\section{MUTUAL INFORMATION IN WEAK- COHERENT-STATE DETECTION USING A HOMODYNE OPTICAL COSTAS LOOP WITH DIFFERENT PHASE ERRORS}

\author{
Josue A. López, ${ }^{1}$ Edith Garcia, ${ }^{2}$ Arturo Arvizu, ${ }^{1}$ \\ Francisco J. Mendieta, ${ }^{3}$ Phillipe Gallion, ${ }^{4}$ and Roberto Conte ${ }^{1}$ \\ ${ }_{1}^{1}$ CICESE Research Center, Carretera Ensenada-Tijuana No. 3918, \\ Ensenada, B.C. 22860, México; Corresponding author: \\ jalopez@cicese.edu.mx \\ ${ }^{2}$ UABC, Calz. Tecnológico 14418, Tijuana, B.C. 22426, México \\ ${ }^{3}$ Agencia Espacial Mexicana, Xola and Universidad, México D.F., \\ México \\ ${ }^{4}$ Telecom-ParisTech, CNRS LTCI UMR 5141, 46 rue Barrault, \\ Paris 75013, France
}

\section{Received 29 October 2012}

ABSTRACT: A free-space experimental set-up for measuring the quadrature components of weak-coherent-state laser signals, based on a homodyne Costas loop configuration is presented. Loop parameters are optimized as a trade-off between quantum and phase noises. Using binary phase-shift-keying modulation, measurements on the mutual information are presented for different photon numbers and phase errors. (C) 2012 Wiley Periodicals, Inc. Microwave Opt Technol Lett 55:769-772, 2013; View this article online at wileyonlinelibrary.com. DOI: $10.1002 /$ mop. 27459

Key words: mutual information; optical Costas loop; weak-coherentstate; homodyne detection

\section{INTRODUCTION}

In communication systems, the main goal is the maximization of the mutual information between the transmitter and the receiver, that is, Alice and Bob in cryptography systems. In optical communications, both with fiber and free space, and particularly those using low-optical power levels (i.e. quantum-level signals), several practical structures for the mutual information maximization have been proposed, to approach the information theoretical limits [1]. Theoretical analysis and experimentation with coherent states are widely reported since they are easily produced with standard stabilized semiconductor laser sources, and the generation of faint signals for quantum levels is easily obtained by strong attenuation of the laser light, leading to weak-coherent-states (WCS) [2, 3].

For the optimum detection of WCS, several configurations have been proposed and experimentally demonstrated, such as the Kennedy and Dolinar receivers, operating in open and closed loops, respectively, approaching the fundamental detection limit; however, these are based on single photon counting [4], which performs poorly at the telecommunications waveband of 1550 $\mathrm{nm}$, in terms of efficiency and speed.

\section{HOMODYNE AND COSTAS LOOP RECEIVERS}

On the other hand, homodyne detection with standard p.i.n. photodetectors has been extensively used in the optical telecommunications waveband, providing the required speed and, for very low-photon numbers, performing better than the Kennedy receiver [4].

Furthermore, the adaptive homodyne detection is linear in the optical-electric field, therefore, many of the results from the communications theory in the radio electric domain could be incorporated, such as error correction and advanced post-processing signal. For example, adaptive homodyne detection has been proposed and experimentally demonstrated for quantum 